\title{
MicroRNAs associated with microvascular invasion in hepatocellular carcinoma and their prognostic impacts in patients undergoing hepatic resection
}

\author{
SUNG KYU SONG $^{1 *}$, MIN GEUN PARK $^{1 *}$, SEUNG-KEUN PARK $^{2}$, CHUL-WOON CHUNG $^{1}$ and YONGKEUN PARK ${ }^{1}$ \\ ${ }^{1}$ Department of Surgery, Catholic Kwandong University International St. Mary's Hospital, \\ Catholic Kwandong University College of Medicine, Incheon 22711; ${ }^{2}$ Department of Supercomputing M\&S \\ Technology Development, Korea Institute of Science and Technology Information, Daejeon 34141, Republic of Korea
}

Received April 8, 2019; Accepted August 30, 2019

DOI: $10.3892 / \mathrm{ol} .2019 .10987$

\begin{abstract}
Although microvascular invasion (McVI) has prognostic value for patients with hepatocellular carcinoma (HCC) who have undergone hepatic resection, few studies have investigated the relationship between McVI and the aberrant expression of microRNAs (miRNAs). The present study identified the miRNAs that were selectively expressed in HCC with $\mathrm{McVI}$ and investigated their prognostic value. Clinical data and the miRNA expression profiles of 372 patients with HCC were extracted from The Cancer Genome Atlas database. miRNAs that were differentially expressed between patients with McVI and those without vascular invasion (VI) were identified and investigated as potential prognostic factors for HCC. The results demonstrated that McVI was a significant predictor of shortened recurrence-free survival (RFS). The 3 year RFS rate in patients with HCC accompanied by McVI was 28.2 and $49.3 \%$ in HCC without VI $(\mathrm{P}<0.001)$. miRNA-141/-582/-9 were upregulated, while miRNA-675 was downregulated in patients with McVI when compared with $\mathrm{HCC}$ patients without VI. $\log _{2}$ fold-changes of miRNA-141/-582/-675/-9 were 0.80 [false discovery rate (FDR), 0.005], 0.55 (FDR, 0.045), -0.99 (FDR, 0.005) and 1.22 (FDR, <0.001), respectively. Kaplan-Meier analysis indicated that the overexpression of miR-141/-582/-9 was significantly associated with poor RFS and a poor overall survival. A text mining analysis revealed that these miRNAs were significantly associated with multifaceted hallmarks of cancer, including
\end{abstract}

Correspondence to: Dr Yongkeun Park, Department of Surgery, Catholic Kwandong University International St. Mary's Hospital, Catholic Kwandong University College of Medicine, 25 Simgokro 100gil, Seo-gu, Incheon 22711, Republic of Korea

E-mail: ykpark@ish.ac.kr

${ }^{*}$ Contributed equally

Key words: hepatectomy, microRNA, recurrence, The Cancer Genome Atlas-Liver Hepatocellular Carcinoma database, vascular invasion 'invasion and metastasis'. In conclusion, the overexpression of miRNA-141/-582/-9 was associated with McVI and a poor survival in patients undergoing hepatic resection for HCC.

\section{Introduction}

Patients with hepatocellular carcinoma (HCC) generally have a poor prognosis even after curative resection. Their 5 year survival and tumor recurrence rates were $30-50 \%$ and $70-85 \%$, respectively, after hepatic resection (1). One reason for these poor outcomes is the tendency for vascular invasion that occurs with HCC (2). As the tumor grows, it invades neighboring vessels via unknown mechanisms. Without vascular invasion, the 5 year survival rates of patients with HCC who have undergone radical hepatic resection are reportedly $50-70 \%$ (3). However, if the tumor invades the adjacent vessels, the prognosis is poor despite curative treatments such as hepatic resection (4). Recently, it has been shown that outcomes after hepatic resection for $\mathrm{HCC}$ can be negatively influenced not only by gross vascular invasion, but also by microvascular invasion (McVI). McVI is defined as a tumor within a vascular space lined by endothelium, which is identified by microscopy (5). However, McVI is difficult to detect with conventional diagnostic tools before surgery. This presents a practical challenge, and many studies have focused on methods for the preoperative prediction of McVI (6).

The mechanism by which HCC tumor cells invade adjacent vessels has yet to be determined; several studies have focused on issues associated with controlling this invasion (7). Recent studies have shown that the epidermal growth factor receptor (EGFR) signaling pathway, Ras, and the Janus kinase/signal transducer and activator of transcription (JAK/STAT) signaling pathway are dysregulated in HCC, promoting cell motility, invasion, and tumor metastasis $(8,9)$. Mounting evidence demonstrates that epithelial-mesenchymal transition (EMT) participates in the aggressive phenotypical changes in cancer cells, and plays a major role in the invasion of cancer cells into adjacent tissues $(10,11)$. Furthermore, several types of small non-coding RNA have been shown to play critical roles in tumorigenesis and tumor progression, with some regulating EMT directly or indirectly, thereby affecting tumor invasion and metastasis $(7,12)$. 
MicroRNAs (miRNAs) are a class of endogenously-expressed small non-coding RNAs. Recent studies have identified clinically significant abnormal patterns of miRNA expression in HCC, with some showing a marked association with aggressive tumor phenotypes or poor survival (13). However, few studies have investigated the relationship between McVI and aberrant miRNA expression. Therefore, the present study aimed to identify miRNAs that act as important contributors to the McVI of HCC.

\section{Materials and methods}

Study data. All molecular data from The Cancer Genome Atlas Liver Hepatocellular Carcinoma database (TCGA LIHC) were downloaded from the data portals (https://tcga-data.nci.nih. gov and http://gdac.broadinstitute.org/), where clinical data are also publicly accessible. Integrated analysis with merged molecular and clinical data is possible because unique TCGA barcode structure is used to identify each molecular sample with the corresponding patient. Information for VI of HCC is written to a variable called 'vascular_invasion' in five different forms: None, Micro, Macro, Not Available, and Unknown. The vascular invasion (VI) status of each tumor sample is segregated into four groups: Absence of VI ('None'), McVI ('Micro'), gross VI ('Macro'), and no available information about VI ('Not Available' or 'Unknown'). The patients, along with their corresponding clinical data and miRNA expression profiles from the database, were categorized into two groups according to the presence or absence of McVI. Patients lacking values regarding vascular invasion were included for the survival analysis of the candidate miRNAs. Descriptive statistics were used to compare patient characteristics including sex, age, etiology, laboratory data, types of surgery, and pathologic data.

miRNA expression data were generated by the Illumina HiSeq 2000 sequencing platform (Illumina, Inc.). Raw read counts were used for the analysis of differentially expressed miRNAs and mRNA. The processed RNA-Seq data, normalized according to the reads per million (RPM) values of miRNAs and mRNAs, were also used in survival and correlation analyses. The present study complied with the publication guidelines provided by TCGA (http://cancergenome.nih.gov/publications/publicationguidelines). All TCGA data are now available without restrictions on their use in publications or presentations.

Screening for differentially expressed miRNAs and prediction of their targets. The differentially expressed sequence (DESeq2) package of the statistics software $\mathrm{R}$ version 3.3.1 was used to assess the differentially expressed miRNAs based on the raw read counts $(14,15)$. Significant differential miRNA expression was determined by false discovery rate (FDR) correction for multiple hypothesis testing (using Benjamini-Hochberg-adjusted P-values) with an FDR threshold $<0.05$. We used a validated target module of the MiRWalk2.0 (zmf.umm.uni-heidelberg.de/mirwalk2) database to identify the target genes of the differentially expressed miRNAs (16).

Statistical analysis. Descriptive statistics of categorical variables focused on frequencies and proportions. All continuous variables were expressed as mean \pm standard deviation. A chi-squared test and t-test were used to compare, respectively, proportions and means. Recurrence-free survival (RFS) and overall survival (OS) were estimated for the two patient groups. The optimal cut-off values for continuous variables of clinical parameters for use in Kaplan-Meier survival analysis were estimated by receiver operating characteristic (ROC) curve analysis. Survival rates and curves were estimated by the Kaplan-Meier method and compared using the log-rank test. The median disease free survival time, median overall survival time, and the $95 \%$ confidence interval were also measured. Cox regression analysis was used to define factors that determined overall and disease-free survival rates by including variables with statistical significance $(\mathrm{P}<0.05)$ for univariate analysis. The statistical analysis was performed using the Epi, GGally, MoonBook, and Survival packages of $\mathrm{R}$ version 3.3.1 (15).

Functional analysis. The Database for Annotation, Visualization, and Integrated Discovery (DAVID) (https://david.ncifcrf.gov/) was used for Kyoto Encyclopedia of Genes and Genome (KEGG) pathway analysis to extract functional pathways involved in the mechanism of McVI. Cancer Hallmarks Analytics Tool (CHAT) was used to determine the association between selective miRNAs and documented evidence of their roles in the hallmarks of cancer (17).

\section{Results}

Patient classification according to vascular invasion status. Five of the 377 HCC patients had no miRNA expression data in the TCGA LIHC database. The remaining 372 patients were categorized into four groups according to their vascular invasion (VI) status: Group A ( $\mathrm{n}=206)$ had tumors without VI; Group B ( $\mathrm{n}=94)$ showed McVI; Group C $(\mathrm{n}=17)$ had gross VI; and Group D ( $\mathrm{n}=55)$ were patients with no available information about VI. The aim of this study was to identify miRNAs selectively expressed in HCC accompanied by McVI compared to $\mathrm{HCC}$ without VI and to investigate their prognostic value. Seventeen patients in Group C were excluded because they had HCC tumors with gross VI (Macro VI). For the tumor nodes metastasis (TNM) stage based on the criteria established by the Liver Cancer Study Group of Japan (LCSGJ), VI is one of three factors for determining the $\mathrm{T}$ stage with tumor size and numbers. According to the Barcelona Clinic for Liver Cancer (BCLC) staging, HCC with gross VI is classified as advanced stage. However, it remains unclear how much microvascular invasion (McVI) provides prognostic information for patients with $\mathrm{HCC}$ from the viewpoint of the extent of tumor invasion or extension. Considered the first step of metastatic dissemination via the vascular route, the prognostic impact of McVI may be intuitively placed between non-VI and gross invasion of vessels. However, there is no strong evidence to support this speculation. Therefore, we decided to exclude patients with HCC accompanied by gross VI (Group C) in our study. Patients in Group D (no available information about VI) were included when conducting univariate survival analysis for each clinicopathological factor and selected miRNA. However, Group D patients were automatically excluded in multivariate survival analysis because they had missing values. 
Table I. Comparison of hepatocellular carcinoma patient clinicopathological data with no vascular invasion (Group A) or with microvascular invasion (Group B).

\begin{tabular}{|c|c|c|c|c|}
\hline Factors & Group A (n=206) & Group B (n=94) & P-value (A vs. B) & Group D $(n=55)$ \\
\hline Sex & & & 0.687 & \\
\hline Male & $136(66.0 \%)$ & $65(69.1 \%)$ & & $42(76.4 \%)$ \\
\hline Female & $70(34.0 \%)$ & $29(30.9 \%)$ & & $13(23.6 \%)$ \\
\hline Age & $59.9 \pm 13.5$ & $58.1 \pm 14.2$ & 0.738 & $59.5 \pm 11.6$ \\
\hline Viral hepatitis & & & 0.097 & \\
\hline No & $81(39.9 \%)$ & $46(51.1 \%)$ & & $19(34.5 \%)$ \\
\hline Yes & $122(60.1 \%)$ & $44(48.9 \%)$ & & $36(65.5 \%)$ \\
\hline History of other malignancy & & & 0.849 & \\
\hline No & $185(89.8 \%)$ & $83(88.3 \%)$ & & $54(98.2 \%)$ \\
\hline Yes & $21(10.2 \%)$ & $11(11.7 \%)$ & & $1(1.8 \%)$ \\
\hline Platelet count $(\mathrm{x} 1,000 / \mu \mathrm{l})$ & $207 \pm 91$ & $229 \pm 118$ & 0.257 & $227 \pm 93$ \\
\hline Serum creatinine (mg/dl) & $0.9 \pm 0.4$ & $1.3 \pm 1.1$ & 0.003 & $0.8 \pm 0.2$ \\
\hline Serum albumin (g/dl) & $3.7 \pm 1.0$ & $4.0 \pm 1.1$ & 0.062 & $3.8 \pm 0.8$ \\
\hline Serum total bilirubin (mg/dl) & $0.8 \pm 0.6$ & $0.9 \pm 0.5$ & 0.450 & $0.6 \pm 0.3$ \\
\hline Child-Turcotte-Pugh classification & & & 0.329 & \\
\hline A & $144(90.0 \%)$ & $58(92.1 \%)$ & & $8(100.0 \%)$ \\
\hline $\mathrm{B}$ or $\mathrm{C}$ & $16(10.0 \%)$ & $5(7.9 \%)$ & & $0(0.0 \%)$ \\
\hline$\alpha$-fetoprotein (ng/ml) & $15,300 \pm 15,700$ & $13,200 \pm 46,800$ & 0.875 & $2,100 \pm 6,300$ \\
\hline Types of hepatic resection & & & $<0.001$ & \\
\hline Major & $133(64.6 \%)$ & $36(39.1 \%)$ & & $21(38.9 \%)$ \\
\hline Minor & $73(35.4 \%)$ & $56(60.9 \%)$ & & $33(61.1 \%)$ \\
\hline Histologic grading by Edmondson and & & & 0.174 & \\
\hline Steiner's classification & & & & \\
\hline $1-2$ & $131(63.9 \%)$ & $51(54.8 \%)$ & & $41(77.4 \%)$ \\
\hline $3-4$ & $74(36.1 \%)$ & $42(45.2 \%)$ & & $12(22.6 \%)$ \\
\hline Residual tumor & & & 0.433 & \\
\hline Negative & $181(96.3 \%)$ & $84(93.3 \%)$ & & $45(93.8 \%)$ \\
\hline Positive & $7(3.7 \%)$ & $6(6.7 \%)$ & & $3(6.2 \%)$ \\
\hline Ishak fibrosis staging system & & & 0.267 & \\
\hline 0 & $52(36.9 \%)$ & $16(28.6 \%)$ & & $1(11.1 \%)$ \\
\hline $1-4$ & $36(25.5 \%)$ & $18(32.1 \%)$ & & $5(55.6 \%)$ \\
\hline $5-6$ & $53(37.6 \%)$ & $22(39.3 \%)$ & & $3(33.3 \%)$ \\
\hline Grade of liver inflammation & & & 0.282 & \\
\hline 0 & $64(46.4 \%)$ & $32(43.2 \%)$ & & $12(92.3 \%)$ \\
\hline $1-2$ & $65(47.1 \%)$ & $33(44.6 \%)$ & & $1(7.7 \%)$ \\
\hline $3-4$ & $9(6.5 \%)$ & $9(12.2 \%)$ & & $0(0.0 \%)$ \\
\hline American Joint Committee on Cancer TNM stage & & & 0.040 & \\
\hline I-II & $164(85.0 \%)$ & $65(73.9 \%)$ & & $21(42.0 \%)$ \\
\hline III-IV & $29(15.0 \%)$ & $23(26.1 \%)$ & & $29(58.0 \%)$ \\
\hline
\end{tabular}

Group A, no vascular invasion; Group B, microvascular invasion without gross vascular invasion; Group D, no available information regarding vascular invasion. The patients with gross vascular invasion (Group C) were excluded from the current study. Mismatches of patient numbers across the variables are due to missing values. TNM, tumor node metastasis.

Table I summarizes the clinical characteristics of the patients in Groups A and B. The patients with McVI (Group B) had significantly higher preoperative serum creatinine levels $(\mathrm{P}=0.003)$; however, the percentage of patients who had undergone major operations was significantly lower $(\mathrm{P}<0.001)$ than those in Group A. There were also notable differences in tumor node metastasis (TNM) stage between the two groups, with patients in Group B exhibiting more advanced stages $(\mathrm{P}<0.04)$. Apart from these differences, the two groups were comparable. The overall median follow-up period was 


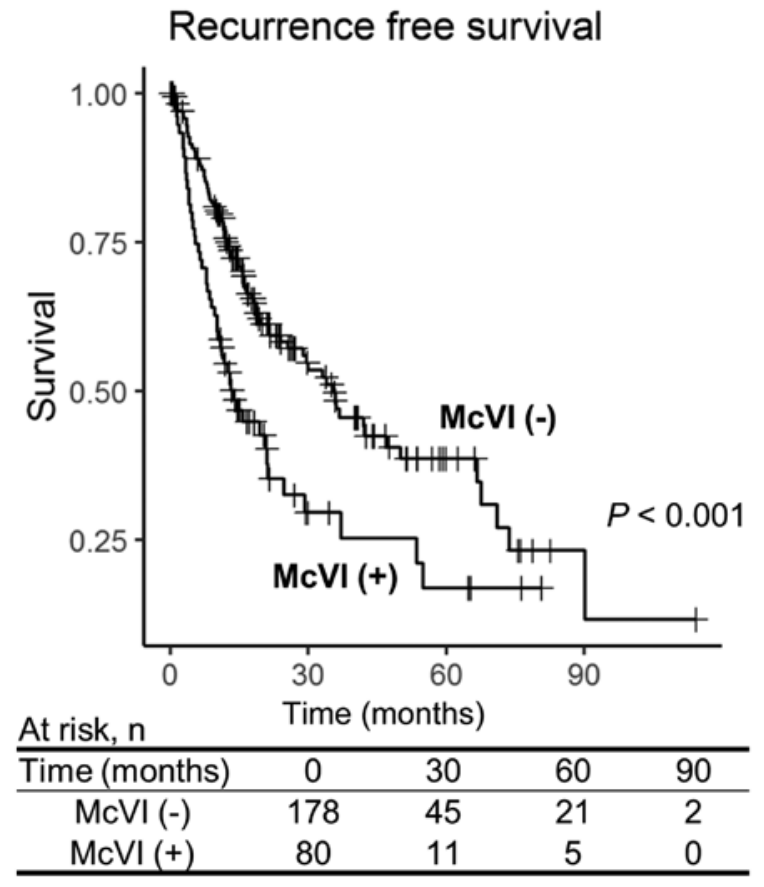

Figure 1. Comparison of recurrence-free survival in patients with microvascular invasion [McVI (+), Group B] and without vascular invasion [McVI (-), Group A]. McVI, microvascular invasion.

13 months (range: 0-114 months). The survival analysis showed significantly worse RFS for the patients with McVI (3 year RFS rates: Group A, 49.3\%; Group B, 28.2\%; P<0.001) (Fig. 1). There was no statistically significant difference in overall survival rate between the groups.

miRNAs were differentially expressed on the basis of the absence or presence of McVI. The expression levels of 540 miRNAs in the 300 samples were obtained from the TCGA expression data. Four miRNAs were differentially expressed between the patients without VI (Group A) and those with McVI (Group B): miRNA-141 [ $\log _{2}$ fold change (FC) of 0.80 and FDR=0.005), miRNA-582 $\left(\log _{2}\right.$ FC of 0.55 and FDR=0.045), and miRNA-9 ( $\log _{2} \mathrm{FC}$ of 1.22 and FDR $<0.001)$ were upregulated to a greater extent in Group B, while miRNA-675 was downregulated to a greater extent in Group B $\left(\log _{2}\right.$ FC of -0.99 and FDR=0.005) (Fig. 2A).

Next, we investigated the discriminatory power of the four miRNAs to assess the presence and the absence of McVI. To evaluate the predictive value, we used the ROC curve to analyze sensitivity and specificity. Each cut-off value was as follows: 2.03 RPM for miRNA-141-3p, 211.98 RPM for miRNA-582-3p, 23.93 RPM for miRNA-675-3p, and 65.37 RPM for miRNA-9-5p. Table II demonstrates the association between the number of aberrantly expressed miRNAs and McVI of resected HCC specimens in 300 patients. Most patients $(90 \%)$ without McVI showed no aberrant expression patterns in these four miRNAs. There were increases in the prevalence of McVI as the number of aberrantly expressed miRNAs increased.

Survival analysis. The ROC-curve-determined optimal cutoff value of miRNA expression in the study participants
(355 patients) was used to classify the patients into underand over-expression groups. The RFS cut-off values for miRNA-141-3p, miRNA-582-3p, miRNA-675-3p, and miRNA-9-5p were 3.34 RPM, 125.79 RPM, 21.15 RPM, and 552.91 RPM, respectively. The OS cut-off value was 4.75 RPM for miRNA-141-3p, 441.09 RPM for miRNA-582-3p, 20.96 RPM for miRNA-675-3p, and 546.69 RPM for miRNA-9-5p. Kaplan-Meier analysis indicated that miRNA-141-3p, miRNA-582-3p, and miRNA-9-5p overexpression was significantly associated with both poor RFS $(\mathrm{P}=0.008$, $\mathrm{P}=0.006$, and $\mathrm{P}=0.013$, respectively) and worse $\mathrm{OS}(\mathrm{P}=0.008$, $\mathrm{P}<0.001$, and $\mathrm{P}<0.001$, respectively) (Fig. 2B). However, HCC patients exhibiting under-expression of miRNA-675 showed no significant difference in survival outcome compared to those with over-expression of miRNA-675 (Tables II and III).

Prediction of associated pathway and functional analysis of selected miRNAs. We identified experimentally validated target genes of miRNA-141-3p, miRNA-582-3p, and miRNA-9-5p using miRNA-mRNA pairs from the MiRWalk2.0 databases (15) There were a total of 459 validated target genes identified for these three selected miRNAs. In order to estimate the regulatory effects, 459 genes were selected for KEGG pathway analysis using the DAVID (https://david.ncifcrf.gov/) functional annotation tool. Twenty-four genes were enriched in microRNAs associated with cancer, while 14 genes were enriched in the FoxO signal pathway (Fig. 3A). A text mining analysis was conducted to determine which 'hallmarks of cancer' were affected when these miRNAs were dysregulated. All of the selected miRNAs were associated with at least six hallmarks of cancer. especially, 'invasion and metastasis,' 'resisting cell death,' 'sustaining proliferative signaling,' and 'tumor promoting inflammation' (Fig. 3B).

\section{Discussion}

In this study, we used publicly available data from the TCGA repositories to identify miRNAs associated with McVI and poor prognosis of HCC. Our results showed that the expression levels of miRNA-141, -582, and -9 were significantly higher in HCC with McVI, while the expression level of miRNA-675 was lower compared to HCC without VI. Furthermore, the overexpression of miRNA-141-3p, -582-3p, and -9-5p was significantly associated with both poor RFS and OS after hepatic resection for HCC. Several recent studies have focused on the relationship between cancer metastasis and the aberrant expression of miRNAs (18). One study showed an association between the decreased expression of miRNA-199a and McVI (19). However, to the best of our knowledge, no previous study has investigated the association between miRNA-141/-582/-675/-9 and McVI in HCC tissue samples.

$\mathrm{McVI}$ is a well-known factor that contributes to tumor recurrence and poor prognosis; its incidence in surgically resected HCC has been reported to be $15-52 \%$ (20). Notably, $\mathrm{McVI}$ was found to be a predictor of early tumor recurrence following hepatic resection for early-stage HCC (21). A recent study showed that its prognostic significance was comparable to that of gross VI limited to segmental or sectional vascular branches of HCC (5). An international consensus conference recommended that patients with $\mathrm{HCC}$ accompanied by McVI 
A

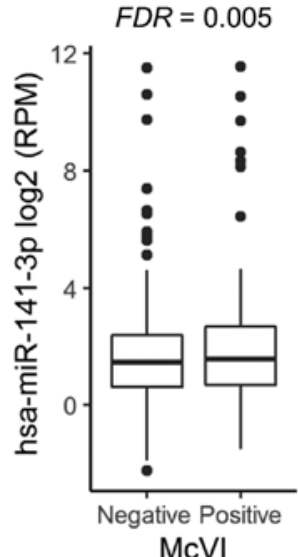

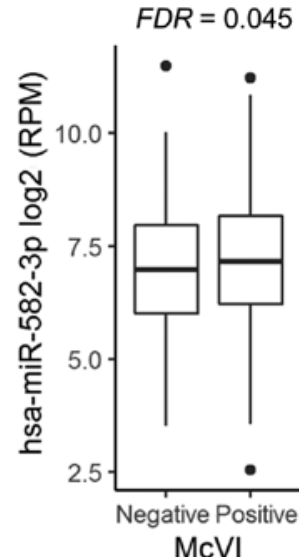
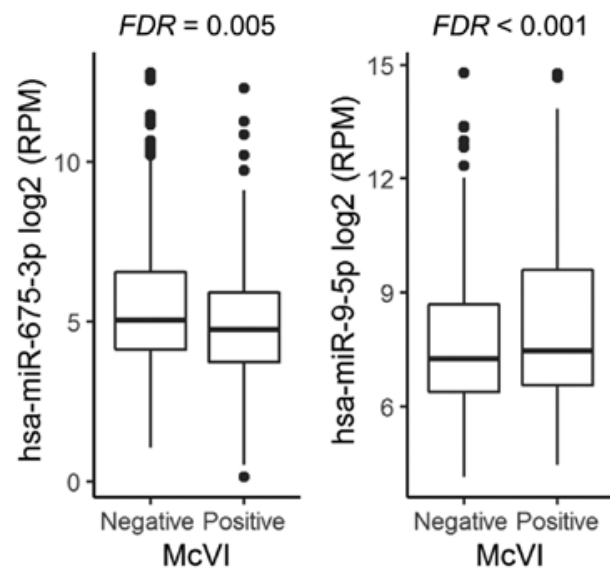

B RFS (miRNA-141-3p)

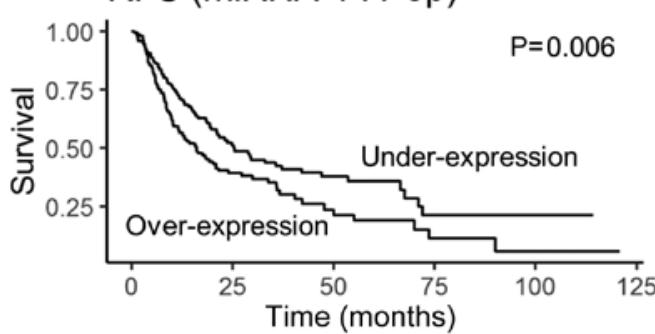

RFS (miRNA-582-3p)

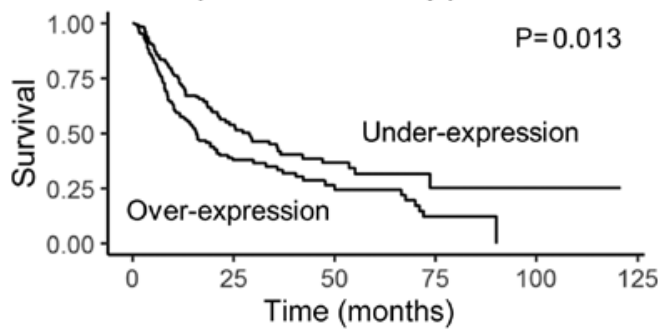

RFS (miRNA-9-5p)
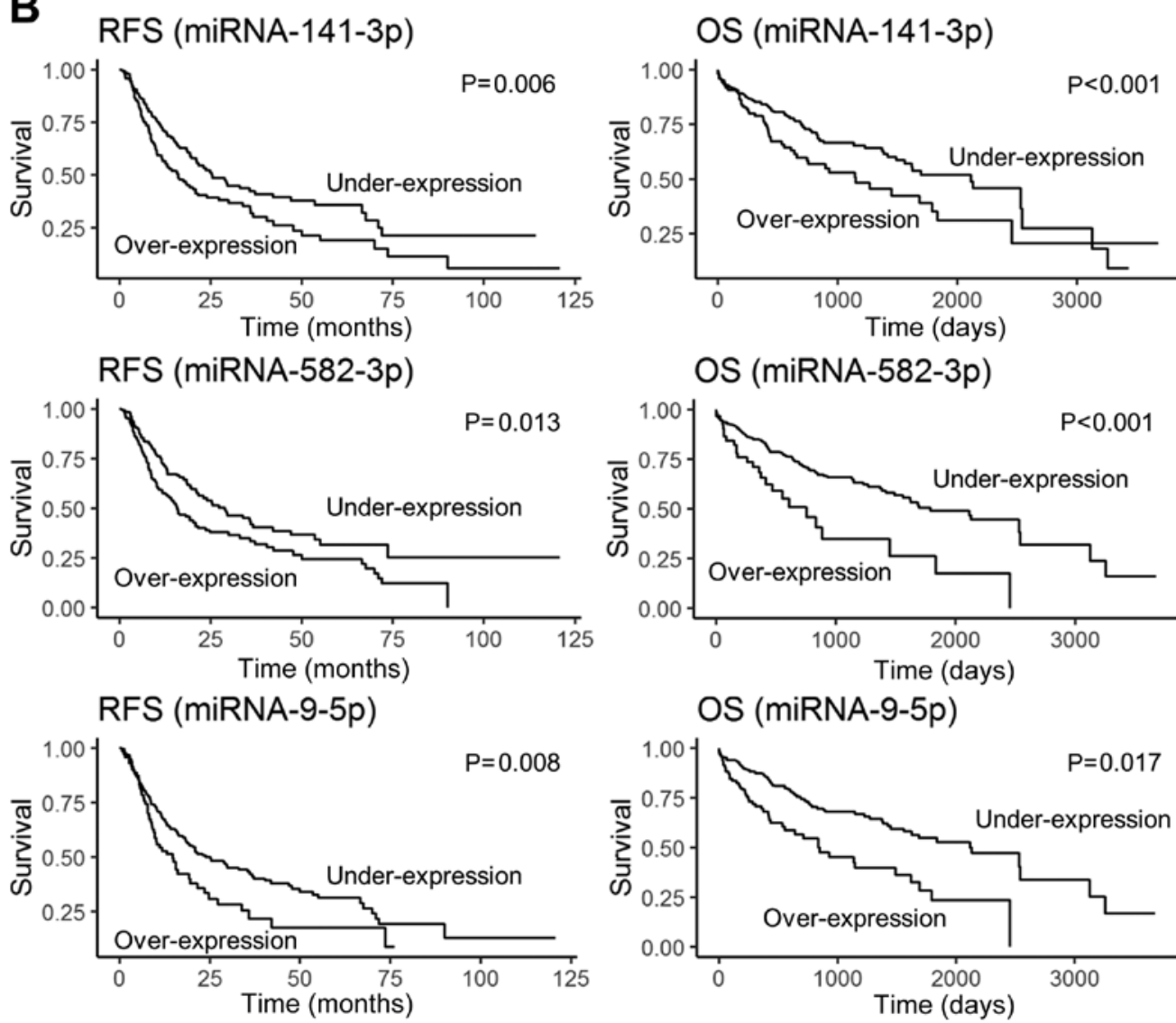

OS (miRNA-582-3p)

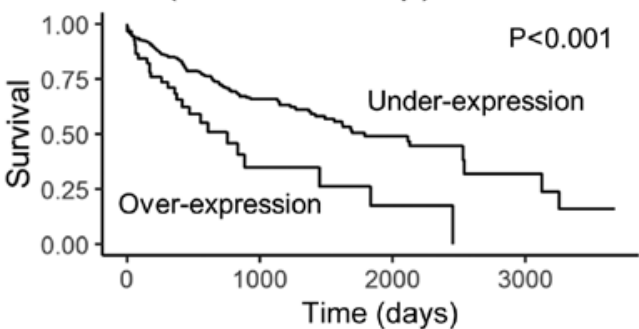

OS (miRNA-9-5p)

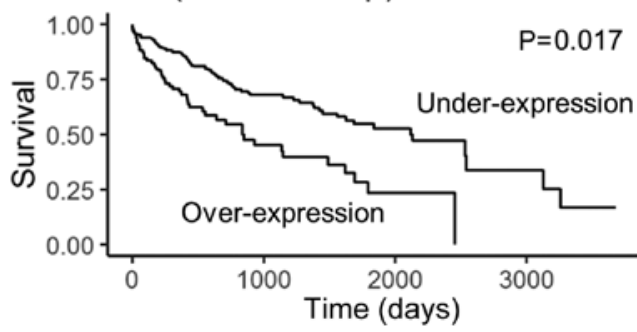

Figure 2. Prognostic impact and expression of selected miRNAs, according to the absence or presence of microvascular invasion (McVI) in HCC tissue. (A) Comparison of miRNA-141-3p, miRNA-582-3p, miRNA-675-3p, and miRNA-9-5p expressions in HCC tissue with McVI or without. (B) RFS and OS curves between upregulated and downregulated miRNA-141-3p, miRNA-582-3p and miRNA-9-5p expression. Under-expression is indicated by expression values less than the cut-off value, while overexpression is indicated by expression values more than the cut-off value. miRNA, microRNA; FDR, false discovery rate; RFS, recurrence-free survival; OS, overall survival; RPM, reads per million.

should not be included as candidates for liver transplantation because of this severely negative oncologic feature (22). For this reason, many studies have focused on how to predict McVI of HCC preoperatively due to the poor sensitivity and specificity of conventional modalities such as positron emission tomography and computed tomography scan for detecting McVI (6).

VI is considered to reflect the aggressiveness of the tumor and is a well-established negative prognostic factor after hepatic resection for HCC (23). However, information is limited regarding the underlying mechanism for VI. One possibility is that VI may be purely coincidental, with high arterial pressure in the tumor resulting in the spread of cancer cells to neighboring vessels. However, recent studies of the mechanism of tumor metastasis have shown the importance of phenotype changes in individual tumor cells $(10,24)$. Genomic data analyses have identified unique genes and non-coding RNAs that play important roles in metastasis $(25,26)$.

miRNAs represent a class of endogenously-expressed small non-coding RNAs, approximately 22 nucleotides in length, that have the capacity to epigenetically regulate gene expression (27). miRNAs primarily function as post-transcriptional regulators through the degradation of their target mRNA or 
Table II. Univariate analysis of factors predictive of recurrence-free survival.

\begin{tabular}{|c|c|c|c|}
\hline Factors & No. of patients & $\operatorname{MDFST}^{\mathrm{a}}(95 \% \mathrm{CI})$ & P-value \\
\hline Sex & & & 0.368 \\
\hline Male & 216 & $21.6(17.6-33.0)$ & \\
\hline Female & 92 & $19.4(14.8-36.7)$ & \\
\hline Age (years) & & & 0.167 \\
\hline$<51$ & 255 & $21.0(17.6-35.8)$ & \\
\hline$\geq 51$ & 53 & $21.2(14.1-33.9)$ & \\
\hline Hepatitis B or C infection status & & & 0.385 \\
\hline Negative & 119 & $19.2(14.9-33.0)$ & \\
\hline Positive & 182 & $23.0(17.6-36.0)$ & \\
\hline Platelet count $(\mathrm{x} 1,000 / \mu \mathrm{l})$ & & & 0.173 \\
\hline$<282$ & 207 & $28.9(21.0-37.1)$ & \\
\hline$\geq 282$ & 48 & $19.6(14.2-55.1)$ & \\
\hline Serum creatinine (mg/dl) & & & 0.077 \\
\hline$<1.1$ & 172 & $21.0(18.3-33.0)$ & \\
\hline$\geq 1.1$ & 78 & $36.0(23.9-73.6)$ & \\
\hline Serum albumin $(\mathrm{g} / \mathrm{dl})$ & & & 0.363 \\
\hline$\geq 4.1$ & 119 & $29.7(21.0-55.1)$ & \\
\hline$<4.1$ & 132 & $20.2(15.7-36.7)$ & \\
\hline Serum total bilirubin $(\mathrm{mg} / \mathrm{dl})$ & & & 0.680 \\
\hline$<0.8$ & 142 & $23.0(19.2-40.4)$ & \\
\hline$\geq 0.8$ & 111 & $29.3(21.0-50.0)$ & \\
\hline Child-Turcotte-Pugh classification & & & 0.604 \\
\hline A & 187 & $23.6(19.2-42.0)$ & \\
\hline $\mathrm{B}$ or $\mathrm{C}$ & 19 & 23.9 (8.6-NA) & \\
\hline$\alpha$-fetoprotein (ng/ml) & & & 0.129 \\
\hline$<85,150$ & 229 & $25.5(19.4-36.0)$ & \\
\hline$\geq 85,150$ & 8 & $30.2(13.1-\mathrm{NA})$ & \\
\hline Extent of resection & & & $<0.001$ \\
\hline Major & 160 & $35.6(23.0-53.5)$ & \\
\hline Minor & 147 & $14.2(10.2-20.9)$ & \\
\hline Residual tumor & & & 0.031 \\
\hline No & 276 & $23.0(18.6-33.9)$ & \\
\hline Yes & 13 & 12.6 (10.8-NA) & \\
\hline Histologic grading by Edmondson and Steiner's classification & & & 0.760 \\
\hline I-II & 195 & $21.6(18.4-29.7)$ & \\
\hline III-IV & 109 & $20.9(12.6-47.0)$ & \\
\hline Ishak fibrosis staging system & & & 0.101 \\
\hline 0 & 57 & 23.9 (15.4-NA) & \\
\hline $1-4$ & 55 & $24.8(18.2-36.7)$ & \\
\hline $5-6$ & 72 & $18.3(12.9-47.0)$ & \\
\hline Grade of liver inflammation & & & 0.950 \\
\hline 0 & 88 & $23.9(20.9-40.4)$ & \\
\hline $1-2$ & 90 & $24.8(18.2-47.0)$ & \\
\hline $3-4$ & 15 & $18.3(9.7-\mathrm{NA})$ & \\
\hline American Joint Committee on Cancer TNM stage & & & 0.005 \\
\hline I-II & 180 & $33.9(24.8-55.1)$ & \\
\hline III-IV & 40 & $13.1(8.6-21.0)$ & \\
\hline MicroRNA-141-3p & & & 0.006 \\
\hline Underexpression & 164 & $25.3(21.0-47.0)$ & \\
\hline Overexpression & 158 & $16.1(11.7-21.6)$ & \\
\hline
\end{tabular}


Table II. Continued.

\begin{tabular}{lcc}
\hline Factors & No. of patients & MDFST $^{\mathrm{a}}(95 \%$ CI $)$ \\
\hline MicroRNA-582-3p & 143 & 0.013 \\
Underexpression & 179 & $28.9(21.2-47.0)$ \\
Overexpression & & $15.7(12.6-21.6)$ \\
MicroRNA-675-3p & 95 & 0.878 \\
Underexpression & 227 & $21.0(16.1-35.6)$ \\
Overexpression & & $(15.7-33.0)$ \\
MicroRNA-9-5p & 247 & 0.008 \\
Underexpression & 75 & $23.9(19.2-36.7)$ \\
Overexpression & $14.8(9.76-23.6)$ \\
\hline
\end{tabular}

${ }^{a}$ Median disease-free survival time (month). Mismatches of patients numbers across the variables occurs due to some missing values. $\mathrm{CI}$, confidence interval; NA, not available.

by inhibiting translation (28). They are emerging as powerful regulators of critical biological processes, including the cell cycle, metabolism, development, and cell differentiation (29). There is also growing evidence that miRNAs are associated with tumor progression, being involved in cell migration, angiogenesis, and invasion $(7,8,19,26)$. miR-141 has been shown to be a prognostic factor for different types of cancer. Recently, miRNA-141 was shown to be involved in controlling cancer cell proliferation of colorectal cancer by regulating the tumor-suppressor gene MAP2K4 (30).

In this study, the upregulation of miRNA-9 and miR-582 was also associated with McVI, as well as a statistically significant difference in survival. miRNA-9 has been shown to be a predictor for different types of cancer. In various studies, high miRNA-9 expression levels were related to worse survival rates and a high risk of cancer metastasis in various carcinomas (31). Another study reported that miR-582 promotes cancer stem cell traits of non-small-cell lung cancer, and that miR-582 inhibition potently inhibits tumor progression (32).

Although this study demonstrated that the downregulation of miRNA-675 was associated with McVI, miRNA-675 expression level was not associated with any statistically significant difference in survival. Previous studies have found that decreased miRNA-675 expression was involved in enhanced cell proliferation and invasion, as well as patient survival, in non-small cell lung cancer and pancreatic cancer $(33,34)$. Its role as a tumor suppressor with anti-oncogenic activity is believed to have emanated from its inhibitory effect on GPR55 or ZEB1. However, other studies have reported differing results. It has been suggested that miRNA-675 and its primary precursor long non-coding RNA H19 play an oncogenic role in gliomas by inhibiting cadherin 13 or retinoblastoma $1(35,36)$. Further studies are necessary to determine the exact roles of miRNA-675 in tumorigenesis and cancer progression.

Lately, there has been a dramatic increase in the number of genomic databases for diseases, including those for cancer. TCGA provides a large volume of publicly available cancer genomic data. Researchers interested in the molecular biology of cancer can access this valuable source of data. In the present study, we used the epigenetic profiles of HCC tumor samples to acquire novel information regarding the role of miRNA-141/-582/-9 in the McVI of HCC. It is likely that, in the near future, newly discovered molecular targets based on the TCGA database will provide clinical applications, including those for the early detection, treatment, and even prevention of cancers. However, the effective translation of cancer genomics or proteomics into clinical practice necessitates progress in analytics; this requires close cooperation between bioinformaticians, mathematicians, and oncologists.

One limitation of the present study is that it was conducted using only one public database. External validation of the findings in this study is currently impossible, as no other genomic database (Gene Expression Omnibus and ArrayExpress) of HCC exists that includes both miRNA profiles and McVI parameters in the clinical data profiles. Furthermore, most clinical and corresponding tissue sample data in the TCGA database come from Western countries. Consequently, further studies with HCC samples from Eastern countries are needed to validate the results of this study. Another drawback of the present study is that it was based on the analysis of secondary data. Consequently, there was a lack of information on important perioperative data, such as liver enzyme profiles, antiviral drug use, and postoperative progression of underlying liver disease, all of which are believed to influence tumor recurrence and de novo malignancy. It is known that miRNAs inhibit target genes through the degradation of their target mRNAs or by inhibiting translation. If a specific miRNA functions by suppressing translation and not through mRNA degradation, it is difficult to identify a target from miRNA and mRNA expression profiles and their correlation analysis alone. In the TCGA database, the protein expression data is limited compared with the miRNA and mRNA data; experimental verification is therefore required.

This study showed that miRNA-141/-582/-9 were overexpressed to a greater extent in HCC tissues with McVI than in those without VI, and that their high expression levels were 
Table III. Univariate analysis of factors predictive of overall survival.

\begin{tabular}{|c|c|c|c|}
\hline Factors & No. of patients & $\operatorname{MOST}^{\mathrm{a}}(95 \% \mathrm{CI})$ & P-value \\
\hline Sex & & & 0.265 \\
\hline Male & 241 & 1836 (1423-NA) & \\
\hline Female & 112 & $1450(887-2532)$ & \\
\hline Ages (years) & & & 0.004 \\
\hline$<70$ & 274 & 2456 (1624-NA) & \\
\hline$\geq 70$ & 79 & $1147(785-1791)$ & \\
\hline Hepatitis B or C infection status & & & 0.013 \\
\hline Negative & 144 & $1135(837-1791)$ & \\
\hline Positive & 202 & 2456 (1685-NA) & \\
\hline Platelet count $(\mathrm{x} 1,000 / \mu 1)$ & & & 0.141 \\
\hline$<282$ & 233 & 2456 (1685-NA) & \\
\hline$\geq 282$ & 55 & $1135(848-2542)$ & \\
\hline Serum creatinine (mg/dl) & & & 0.746 \\
\hline$<0.78$ & 80 & 1372 (887-NA) & \\
\hline$\geq 0.78$ & 203 & 2456 (1694-NA) & \\
\hline Serum albumin $(\mathrm{g} / \mathrm{dl})$ & & & 0.419 \\
\hline$<4.3$ & 85 & 3258 (1560-NA) & \\
\hline$\geq 4.3$ & 198 & 1694 (1423-NA) & \\
\hline Serum total bilirubin $(\mathrm{mg} / \mathrm{dl})$ & & & 0.144 \\
\hline$<1$ & 201 & $2131(1624-3258)$ & \\
\hline$\geq 1$ & 84 & NA (1622-NA) & \\
\hline Child-Turcotte-Pugh classification & & & 0.911 \\
\hline A & 209 & 2542 (2131-NA) & \\
\hline $\mathrm{B}$ or $\mathrm{C}$ & 20 & 987 (601-NA) & \\
\hline$\alpha$-fetoprotein (ng/ml) & & & 0.086 \\
\hline$<85,150$ & 259 & 2456 (1685-NA) & \\
\hline$\geq 85,150$ & 8 & NA (633-NA) & \\
\hline Extent of resection & & & 0.061 \\
\hline Major & 189 & 2116 (1624-NA) & \\
\hline Minor & 161 & 1149 (887-NA) & \\
\hline Residual tumor & & & 0.113 \\
\hline No & 309 & $1791(1450-3125)$ & \\
\hline Yes & 16 & 837 (385-NA) & \\
\hline Histologic grading by Edmondson and Steiner's classification & & & 0.224 \\
\hline I-II & 222 & $1791(1423-2542)$ & \\
\hline III-IV & 127 & NA (1149-NA) & \\
\hline Ishak fibrosis staging system & & & 0.913 \\
\hline 0 & 69 & 2456 (931-NA) & \\
\hline $1-4$ & 59 & 1791 (1372-NA) & \\
\hline $5-6$ & 77 & NA (1685-NA) & \\
\hline Grade of liver inflammation & & & 0.133 \\
\hline 0 & 108 & 2456 (1624-NA) & \\
\hline $1-2$ & 99 & NA (1368-NA) & \\
\hline $3-4$ & 17 & NA (359-27.9) & \\
\hline American Joint Committee on Cancer TNM stage & & & 0.001 \\
\hline I-II & 180 & NA (2456-NA) & \\
\hline III-IV & 40 & 1791 (660-NA) & \\
\hline MicroRNA-141-3p & & & $<0.001$ \\
\hline Underexpression & 240 & 2116 (1560-NA) & \\
\hline Overexpression & 130 & $1149(743-1836)$ & \\
\hline
\end{tabular}


Table III. Continued.

\begin{tabular}{lcc}
\hline Factors & No. of patients & MOST (95\% CI) \\
\hline MicroRNA-582-3p & 312 & $1791(1490-3258)$ \\
Underexpression & 58 & $757(415-\mathrm{NA})$ \\
Overexpression & & $1271(837-\mathrm{NA})$ \\
MicroRNA-675-3p & 113 & 0.172 \\
Underexpression & 257 & $2116(1423-\mathrm{NA})$ \\
Overexpression & & 0.017 \\
MicroRNA-9-5p & 274 & $2116(1560-\mathrm{NA})$ \\
Underexpression & 96 & $837(558-1694)$ \\
Overexpression & & 1601 \\
\hline
\end{tabular}

${ }^{a}$ Median overall survival time (day). Mismatches of patients numbers across the variables occurs due to some missing values. CI, confidence interval; NA, not available.

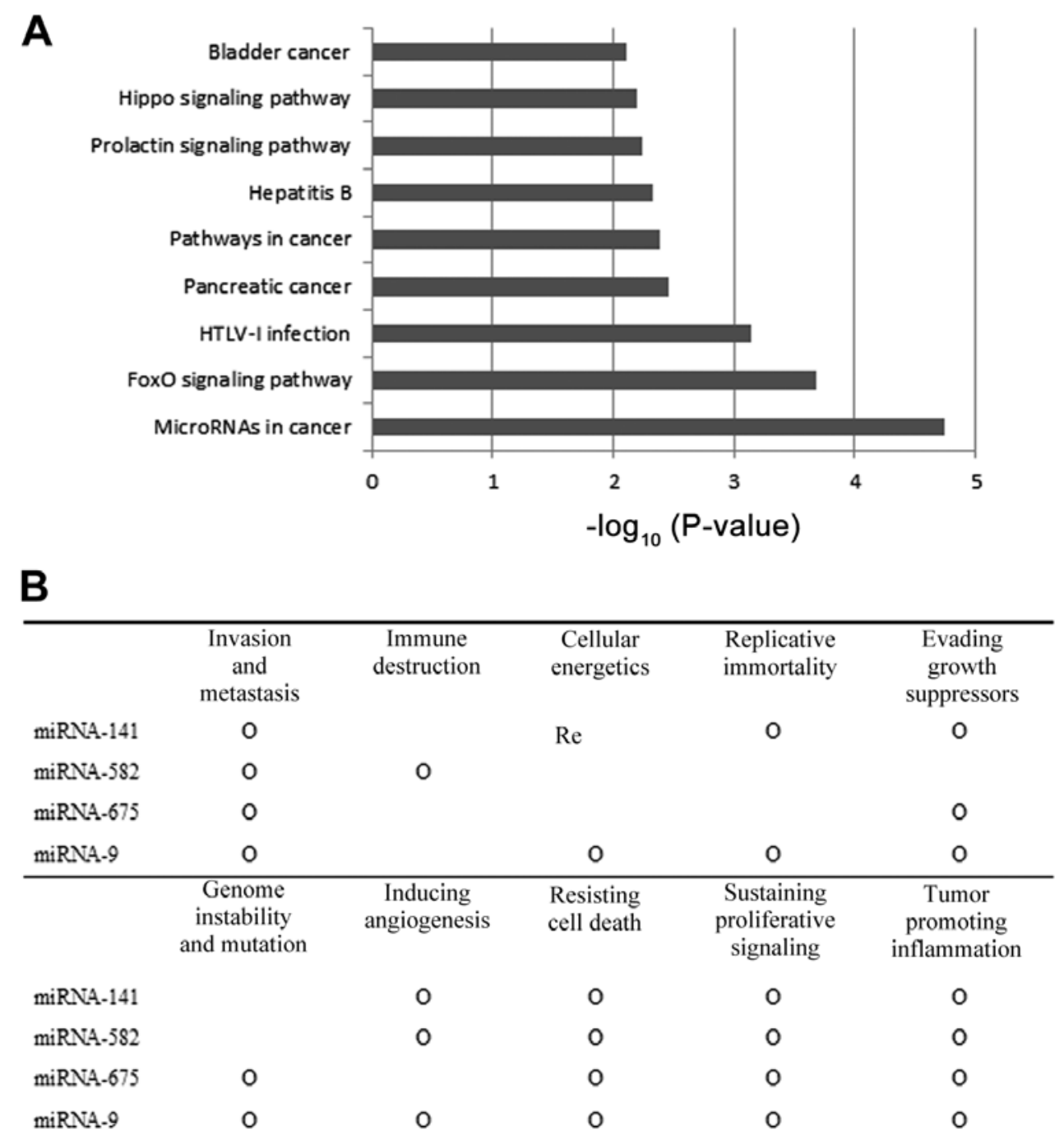

Figure 3. Gene Ontology and CHAT analysis of selected miRNAs. (A) Significantly enriched pathways of miRNA-141-3p, miRNA-582-3p and miRNA-9-5p. (B) Summary of CHAT analysis for miRNA-141, miRNA-582, miRNA-675 and miRNA-9. miRNA, microRNA; CHAT, Cancer Hallmarks Analytics Tool; HTLV-1, human T-lymphotropic virus-1.

significantly associated with poor survival of patients after hepatic resection for HCC. Therefore, these three miRNAs warrant consideration as potential therapeutic targets for cancer treatment. 


\section{Acknowledgements}

Not applicable.

\section{Funding}

The present study was supported by the National Research Foundation of Korea (NRF) grant funded by the Korea government (MSIP; Ministry of Science, ICT \& Future Planning; grant nos. 2017R1C1B1008436 and 2017R1C1B5076680).

\section{Availability of data and materials}

The datasets generated and analyzed during the current study are available in The Cancer Genome Atlas Liver Hepatocellular Carcinoma database (TCGA LIHC) repository, https://tcga-data. nci.nih.gov and http://gdac.broadinstitute.org/.

\section{Authors' contributions}

YP, SKS and MGP designed the present study. YP, SKS, MGP, SKP and CWC analyzed and interpreted the data. YP and SKS wrote and revised the manuscript. MGP, SKP and CWC reviewed the manuscript. All authors discussed the study and reviewed the manuscript. All authors read and approved the final manuscript.

\section{Ethics approval and consent to participate}

Not applicable.

\section{Patient consent for publication}

Not applicable.

\section{Competing interests}

The authors declare that they have no competing interests.

\section{References}

1. Llovet JM, Schwartz M and Mazzaferro V: Resection and liver transplantation for hepatocellular carcinoma. Semin Liver Dis 25: 181-200, 2005.

2. Thuluvath PJ: Vascular invasion is the most important predictor of survival in HCC, but how do we find it? J Clin Gastroenterol 43: 101-102, 2009.

3. Llovet JM, Bru C and Bruix J: Prognosis of hepatocellular carcinoma: The BCLC staging classification. Semin Liver Dis 19: 329-338, 1999.

4. Peng ZW, Guo RP, Zhang YJ, Lin XJ, Chen MS and Lau WY: Hepatic resection versus transcatheter arterial chemoembolization for the treatment of hepatocellular carcinoma with portal vein tumor thrombus. Cancer 118: 4725-4736, 2012.

5. Park YK, Song SK, Kim BW, Park SK, Chung CW and Wang HJ: Prognostic significance of microvascular invasion in tumor stage for hepatocellular carcinoma. World J Surg Oncol 15: 225, 2017.

6. Shirabe K, Toshima T, Kimura K, Yamashita Y, Ikeda T, Ikegami T, Yoshizumi T, Abe K, Aishima S and Maehara Y: New scoring system for prediction of microvascular invasion in patients with hepatocellular carcinoma. Liver Int 34: 937-941, 2014.

7. Wang W, Lin H, Zhou L, Zhu Q, Gao S, Xie H, Liu Z, Xu Z, Wei J, Huang X and Zheng S: MicroRNA-30a-3p inhibits tumor proliferation, invasiveness and metastasis and is downregulated in hepatocellular carcinoma. Eur J Surg Oncol 40: 1586-1594, 2014.
8. Dang Z, Shangguan J, Zhang C, Hu P, Ren Y, Lv Z, Xiang H and Wang X: Loss of protocadherin-17 (PCDH-17) promotes metastasis and invasion through hyperactivation of EGFR/MEK/ERK signaling pathway in hepatocellular carcinoma. Tumour Biol 37: 2527-2535, 2016.

9. Calvisi DF, Ladu S, Gorden A, Farina M, Conner EA, Lee JS, Factor VM and Thorgeirsson SS: Ubiquitous activation of Ras and Jak/Stat pathways in human HCC. Gastroenterol 130: 1117-1128, 2006.

10. Bacigalupo ML, Manzi M, Espelt MV, Gentilini LD, Compagno D, Laderach DJ, Wolfenstein-Todel C, Rabinovich GA and Troncoso MF: Galectin-1 triggers epithelial-mesenchymal transition in human hepatocellular carcinoma cells. J Cell Physiol 230: 1298-1309, 2015.

11. Wang CH, Guo ZY, Chen ZT, Zhi XT, Li DK, Dong ZR, Chen ZQ, Hu SY and Li T: TMPRSS4 facilitates epithelial-mesenchymal transition of hepatocellular carcinoma and is a predictive marker for poor prognosis of patients after curative resection. Sci Rep 5: 12366, 2015.

12. Li PF, Chen SC, Xia T, Jiang XM, Shao YF, Xiao BX and Guo JM: Non-coding RNAs and gastric cancer. World J Gastroenterol 20: 5411-5419, 2014.

13. Li L, Liu Y, Guo Y, Liu B, Zhao Y, Li P, Song F, Zheng H, Yu J, Song T, et al: Regulatory MiR-148a-ACVR1/BMP circuit defines a cancer stem cell-like aggressive subtype of hepatocellular carcinoma. Hepatology 61: 574-584, 2015.

14. Love MI, Huber W and Anders S: Moderated estimation of fold change and dispersion for RNA-seq data with DESeq2. Genome Biol 15: 550, 2014.

15. R Core Team: R: A language and environment for statistical computing. R Foundation for Statistical Computing, Vienna, 2016. https://www.R-project.org/. Assessed April 1, 2018.

16. Dweep H and Gretz N: MiRWalk2.0: A comprehensive atlas of microRNA-target interactions. Nat Methods 12: 697, 2015.

17. Baker S, Ali I, Silins I, Pyysalo S, Guo Y, Hogberg J, Stenius U and Korhonen A: Cancer hallmarks analytics tool (CHAT): A text mining approach to organize and evaluate scientific literature on cancer. Bioinformatics 33: 3973-3981, 2017.

18. Fang F, Chang RM, Yu L, Lei X, Xiao S, Yang H and Yang LY: MicroRNA-188-5p suppresses tumor cell proliferation and metastasis by directly targeting FGF5 in hepatocellular carcinoma. J Hepatol 63: 874-885, 2015.

19. Shi Y, Song Q, Yu S, Hu D and Zhuang X: Microvascular invasion in hepatocellular carcinoma overexpression promotes cell proliferation and inhibits cell apoptosis of hepatocellular carcinoma via inhibiting miR-199a expression. Onco Targets Ther 8: 2303-2310, 2015.

20. Rodríguez-Perálvarez M, Luong TV, Andreana L, Meyer T, Dhillon AP and Burroughs AK: A systematic review of microvascular invasion in hepatocellular carcinoma: Diagnostic and prognostic variability. Ann Surg Oncol 20: 325-339, 2013.

21. Park YK, Song SK, Kim BW, Park SK, Lee JI, Lim SS and Wang HJ: Conditional survival analysis demonstrates that recurrence risk of surgically treated hepatocellular carcinoma evolves with time. J Gastrointest Surg 21: 1237-1244, 2017.

22. Clavien PA, Lesurtel M, Bossuyt PM, Gores GJ, Langer B and Perrier A; OLT for HCCConsensus Group: OLT for HCC consensus group. Recommendations for liver transplantation for hepatocellular carcinoma: An international consensus conference report. Lancet Oncol 13: e11-e22, 2012.

23. Bai T, Chen J, Xie ZB, Wu FX, Wang SD, Liu JJ and Li LQ: The efficacy and safety of postoperative adjuvant transarterial embolization and radiotherapy in hepatocellular carcinoma patients with portal vein tumor thrombus. Onco Targets Ther 9: 3841-3848, 2016.

24. Guo HB, Zhang Y and Chen HL: Relationship between metastasis-associated phenotypes and N-glycan structure of surface glycoproteins in human hepatocarcinoma cells. J Cancer Res Clin Oncol 127: 231-236, 2001.

25. Hu X, Zhao Y, Wei L, Zhu B, Song D, Wang J, Yu L and Wu J: CCDC178 promotes hepatocellular carcinoma metastasis through modulation of anoikis. Oncogene 36: 4047-4059, 2017.

26. Budhu A, Jia HL, Forgues M, Liu CG, Goldstein D, Lam A, Zanetti KA, Ye QH, Qin LX, Croce CM, et al: Identification of metastasis-related microRNAs in hepatocellular carcinoma. Hepatology 47: 897-907, 2008.

27. Iyengar BR, Choudhary A, Sarangdhar MA, Venkatesh KV, Gadgil CJ and Pillai B: Non-coding RNA interact to regulate neuronal development and function. Front cell Neurosci 8: 47, 2014. 
28. Filipowicz W, Jaskiewicz L, Kolb FA and Pillai RS Post-transcriptional gene silencing by siRNAs and miRNAs. Curr Opin Struct Biol 15: 331-341, 2005.

29. Cora D, Re A, Caselle M and Bussolino F: MicroRNA-mediated regulatory circuits: Outlok and perspectives. Phys Biol 14: 045001, 2017.

30. Ding L, Yu LL, Han N and Zhang BT: MiR-141 promotes colon cancer cell proliferation by inhibiting MAP2K4. Oncol Lett 13: 1665-1671, 2017.

31. Zhang Y, Zhou J, Sun M, Sun G, Cao Y, Zhang H, Tian R, Zhou L, Duan L, Chen X and Lun L: Prognostic value of microRNA-9 in various cancers: A meta-analysis. Pathol Oncol Res 23: 573-582, 2017.

32. Fang L, Cai J, Chen B, Wu S, Li R, Xu X, Yang Y, Guan H, Zhu X, Zhang L, et al: Aberrantly expressed miR-582-3p maintains lung cancer stem cell-like traits by activating Wnt/beta-catenin signalling. Nat Commun 6: 8640, 2015.
33. He D, Wang J, Zhang C, Shan B, Deng X, Li B, Zhou Y, Chen W, Hong J, Gao Y, et al: Down-regulation of miR-675-5p contributes to tumor progression and development by targeting pro-tumorigenic GPR55 in non-small cell lung cancer. Mol Cancer 14: 73, 2015.

34. Ma L, Tian X, Guo H, Zhang Z, Du C, Wang F, Xie X, Gao H, Zhuang Y, Kornmann M, et al: Long noncoding RNA H19 derived miR-675 regulates cell proliferation by down-regulating E2F-1 in human pancreatic ductal adenocarcinoma. J Cancer 9: 389-399, 2018.

35. Shi Y, Wang Y, Luan W, Wang P, Tao T, Zhang J, Qian J, Liu N and You Y: Long non-coding RNA H19 promotes glioma cell invasion by deriving miR-675. PLoS One 9: e86295, 2014.

36. Zheng Y, Lu X, Xu L, Chen Z, Li Q and Yuan J: MicroRNA-675 promotes glioma cell proliferation and motility by negatively regulating retinoblastoma 1. Hum Pathol 69: 63-71, 2017. 